\title{
Mapping Software Metrics to Module Complexity: A Pattern Classification Approach
}

\author{
Nick John Pizzi
}

Department of Computer Science, University of Manitoba, Winnipeg, Canada. Email: pizzi@cs.umanitoba.ca

Received June $3^{\text {rd }}, 2011$; revised June $30^{\text {th }}, 2011$; accepted July $1^{\text {st }}, 2011$.

\begin{abstract}
A desirable software engineering goal is the prediction of software module complexity (a qualitative concept) using automatically generated software metrics (quantitative measurements). This goal may be couched in the language of pattern classification; namely, given a set of metrics (a pattern) for a software module, predict the class (level of complexity) to which the module belongs. To find this mapping from metrics to complexity, we present a classification strategy, stochastic metric selection, to determine the subset of software metrics that yields the greatest predictive power with respect to module complexity. We demonstrate the effectiveness of this strategy by empirically evaluating it using a publicly available dataset of metrics compiled from a medical imaging system and comparing the prediction results against several classification system benchmarks.
\end{abstract}

Keywords: Software Metrics, Pattern Classification, Feature Selection, Software Complexity

\section{Introduction}

Software systems are used to solve or model increasingly complex problems in a variety of application domains. However, problem complexity should not manifest itself as software module complexity. That is, problem complexity should only produce software complexity as an emergent property and not as an intrinsic property of individual software modules (functions, objects, templates, and so on). Intrinsic module complexity is an undesirable characteristic that is often subjectively identified by software developers for some form of remedial action. However, this subjective process is slow, tedious, and error-prone. Automating (to some extent) this process would be desirable especially if coupled with a more rigorous quantitative approach. One possible strategy is the prediction of software module complexity using automatically generated quantitative measurements, namely software metrics $[1,2]$.

A software metric is a mapping from a software module to a set of numerical values to quantify one or more software attributes [3]. These metrics are commonly regarded as important factors reflecting the nature of software systems including, among other characteristics, the property of complexity [4-7]. Software complexity has a direct influence on its quality, clarity, extensibility, and maintainability. The analysis of complexity may be cast as a problem of pattern classification: find a mapping that predicts the class label (level of software complexity) of a pattern (software module), given only its features (software metrics). Such a classification system could serve as a "complexity filter" for software modules. For instance, a project manager or software developer could use such a filter to predict module complexity to identify highly complex software modules for review and possible revision.

While some metrics may have discriminatory power, that is, predictive utility vis-à-vis complexity, other metrics may have none. Moreover, it is possible for some metrics to have a potential deleterious effect on the classification process [8-11]; these confounding metrics distort class boundaries in the metric space (for instance, delineating between high complexity and low complexity modules), thereby reducing classification performance (accuracy). Therefore, it is important not only to identify discriminatory metrics but to also cull confounding ones. To this end, stochastic metric selection is investigated as a pattern classification strategy to determine the subset of software metrics that best predict software module complexity. The strategy, which stochastically examines subsets of software metrics for their predictive power, is empirically evaluated using a database of software metrics from a large medical image processing system, and 
the strategy's classification performance is compared against several classification system benchmarks. Section 2 presents a brief overview of the dataset's software metrics. Section 3 describes the classification strategy for finding a mapping between software metrics and module complexity. Details relating to the design of the experiments and the results compared against the benchmarks are presented in Section 4 and Section 5, respectively, with concluding remarks in Section 6.

\section{Classification \& Software Measurements}

Couched in classification terms, we are interested in finding a mapping between patterns (metrics) and class labels (complexity); formally, $\boldsymbol{X}=\left\{\left(\boldsymbol{x}_{k}, \omega_{k}\right), k=1,2, \cdots, N\right\}$ is a set of $N$ patterns, $\boldsymbol{x}_{k} \in \mathfrak{R}^{n}$, with respective class labels, $\omega_{k} \in \Omega=\{1,2, \cdots, c\} . \boldsymbol{X}$ is randomly allocated to a design subset (often referred to as a training set), $\boldsymbol{X}^{D}$, comprising $N^{D}$ patterns, or a validation subset (often referred to as a test set), $\boldsymbol{X}^{V}$, comprising $N^{V}$ patterns $\left(N^{D}+N^{V}=N\right)$. A classification system finds a mapping, $f: \boldsymbol{X}^{D} \rightarrow \Omega$. Subsequently, $f$ is validated using $\boldsymbol{X}^{V}, f: \boldsymbol{X}^{V} \rightarrow \Omega$. In our specific case, $\boldsymbol{X}$ is the dataset described in Section 2.1 where $\boldsymbol{x}_{k}$ is the list of software metrics (see Section 2.2) for software module $k$. As defined in Section 2.3, we will use $c=2$ class labels for complexity: low, $\omega_{1}$, and high, $\omega_{2}$.

It is important to note at this point that the choices for metrics and class labels described below were made for the sake of expediency; specifically, these were the parameters that were supplied in the publicly available dataset. Class labels, based on change counts (see Section 2.3), are unlikely to be the best delineators of software module complexity, nor are the supplied metrics necessarily the best predictors of complexity. No claim is made as to the optimality of these parameters vis-à-vis complexity prediction, rather we make the simple pragmatic assertion that they will serve as an experimental test bed to examine the efficacy of treating the assessment of software module complexity as a problem of pattern classification.

\subsection{Medical Imaging System}

This investigation considers the Medical Imaging System (MIS) dataset [12] that has been used in some other studies dealing with software quality $[13,14]$. MIS is a commercial software system consisting of approximately 4500 modules and comprising approximately 400000 lines of code. The dataset consists of $N=390$ software modules. Each module is described using $n=11$ software metrics as well as its change count, which is a reflection of its complexity [7].

\subsection{Software Metrics}

As the software engineering literature provides thorough and extensive discussions of the software metrics used in this investigation, including their relative advantages and disadvantages [13,15-17], we restrict ourselves to brief summaries. The first software metrics collected for each MIS module are the ubiquitous "lines of code" (L1), which is the total number of lines of code including programmer comments, and the number of lines of code excluding comments (L2). The next set of software measures includes the number of code characters (C1), comments (C2), comment characters (C3), and code characters (C4), respectively.

Another set of software measures pertain to more complex computations based on the length of a program: Halstead's measure of program length (P1), which is the sum of the total number of program operators and the total number of program operands [18]; Halstead's software measure of estimated program length (P2), P2 = $\eta_{1} \log \eta_{1}+\eta_{2} \log \eta_{2}$, where $\eta_{1}$ and $\eta_{2}$ are the total number of unique program operators and program operands, respectively; and, Jensen's program length (P3), P3 = $\left(\log \eta_{1}\right) !+\left(\log \eta_{2}\right) ![16]$.

The final two software measures concern program control flow graphs: McCabe's cyclomatic number (F1), which is one more than the total number of decision nodes in the control flow graph [19]; and Belady's bandwidth metric $(\mathrm{F} 2), \mathrm{F} 2=\mathrm{a}^{-1} \sum_{i}\left(i \times a_{i}\right)$, where $a_{i}$ is the number of nodes at level $i$ in a nested control flow graph of a nodes.

\subsection{Change Count}

In this investigation, the change count (CC) measure is used as the class label. While the MIS dataset has 42 unique CC labels, it is unrealistic to expect reliable classification results using so many class labels with only 390 modules. As a result, the $\mathrm{CC}$ class labels are aggregated into two qualitative classes, low $\left(\omega_{1}\right)$ complexity versus high $\left(\omega_{2}\right)$ complexity, using two different points of delineation, DS3 (no more than three programmer changes) and DS4 (no more than four programmer changes). The delineations were arbitrarily chosen, before the classification experiments were conducted, so that the two complexity classes were well distributed (roughly an equal number of software modules in each class).

With DS3, the number of software modules in the low complexity class is $N_{11}=192$ and the number of software modules in the high class is $N_{12}=198$, respectively. With DS4, the number of software modules in the low class is $N_{21}=220$ and the number of modules in the high class is $N_{22}=170$.

\section{Classification Method}

\subsection{Stochastic Metric Selection}

The motivation for pre-processing strategies exploiting 
pattern feature selection [20-22] is to simplify the determination and construction of optimal class boundaries in the feature space. An example of such a strategy is stochastic metric selection (SMS), which is based on previous work described in [11] but modified to deal with software metrics. Figure 1 presents a flowchart of the iterative SMS algorithm, which individual steps we now describe below.

The investigator must select the classifier types to be used; SMS operates with homogeneous or heterogeneous collections of pattern classifiers e.g., linear discriminant analysis, artificial neural networks, genetic algorithms, and support vector machines). The algorithm terminates upon exceeding a maximum number of iterations, $\tau$, or a performance (classification accuracy) threshold, $P_{S}$. A number of different performance measures may be used (see Section 4.2). The subsets of metrics are constrained by four parameters: the minimum and maximum number of metric subsets, and the minimum and maximum cardinality for a metric subset. Metric subsets may also be quadratically combined to produce "new" metrics (see below). Using $b$-fold validation, $\boldsymbol{X}$ is randomly allocated to either $\boldsymbol{X}^{D}$ or $\boldsymbol{X}^{V}$. During the design phase, an instantiation of one of the pattern classifier types is used with randomly sampled metric subsets (satisfying the constraints above) from $\boldsymbol{X}^{D}$ to design a mapping. Subsequently, during the validation phase, the classification performance (accuracy), $P$, is measured using $\boldsymbol{X}^{V}$. This process iterates with many pairs of classifier instances and metric subsets.

SMS exploits the quadratic combination of metric subsets. The intent is that if the original metric space had non-linear class boundaries, the new (quadratic) parameter space may end up having class boundaries that are more linear. SMS has three categories of quadratic combinations: using the original metric subset; squaring the

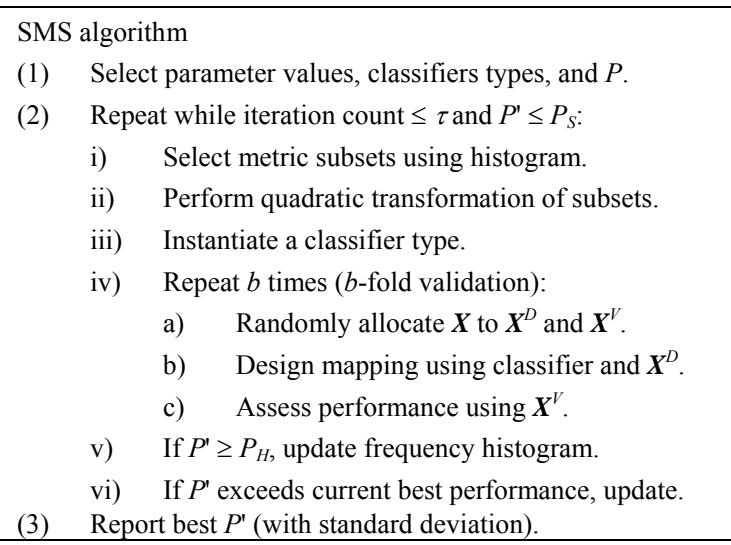

Figure 1. Flowchart for SMS algorithm $\left(P^{\prime}\right.$ is the median performance result of a set of $b$ runs). metric values for a particular metric subset; and using all pair-wise cross products of metrics from two subsets. The probabilities (set by the investigator) of selecting one of these quadratic metric combination categories must sum to 1 .

The stochastic nature of SMS is controlled by a metric frequency histogram whereby the performance of each classification task is assessed using the selected performance measure. If the performance exceeds the histogram fitness threshold, $P_{H}$, the histogram is incremented at those metric indices corresponding to the subsets used by the classification task. This metric histogram is used to generate an ad hoc cumulative distribution function, which is used when randomly sampling new metric subsets. So, rather than each metric having an equal likelihood of being selected for a new classification task (a new classifier instance), those software metrics that were used in previous "successful" classification tasks have a greater likelihood of being chosen. A temperature term, 0 $\leq t \leq 1$, provides additional control over this metric sampling process. If $t=0$, the cumulative distribution function is used but, as $t \rightarrow 1$, the randomness becomes more uniform (when $t=1$ a strict uniform distribution is used).

\subsection{Underlying Classifier}

As previously mentioned, the SMS algorithm can operate with any kind of underlying pattern classifier. In this investigation, we chose a simple linear pattern classifier using linear discriminant analysis (LDA) [8], which determines linear class boundaries between $c$ classes (in our case, complexity) while simultaneously taking into account between-class and within-class variances. If the error distributions for each class are the same, it can be shown that LDA finds the optimal set of linear class boundaries. In real-world pattern classification situations, this optimality is seldom achieved since different classes of patterns typically give rise to different (sometimes, significantly different) distributions.

The LDA algorithm allocates a pattern, $\boldsymbol{x}$, to class $k$ for which the probability distribution, $p_{k}(\boldsymbol{x})$, is greatest, that is, $\boldsymbol{x}$ is allocated to class $k$, if $q_{k} p_{k}(\boldsymbol{x}) \geq q_{i} p_{i}(\boldsymbol{x})(\forall i \neq k)$, where $q_{k}$ are the prior (or proportional) probabilities. $L_{k}(\boldsymbol{x})$ $=\log q_{k}+m_{k}{ }^{\mathrm{T}} \boldsymbol{W}^{-1}\left(\boldsymbol{x}-1 / 2 \boldsymbol{m}_{k}\right)$ is the discriminant function for class $k$ where $\boldsymbol{m}_{k}$ is the mean for class $k$ and $\boldsymbol{W}$ is the covariance matrix. The metric space hyperplane, $F_{k i}(x)$, separating class $k$ from class $i$ is defined as $F_{k i}(\boldsymbol{x})=L_{k}(\boldsymbol{x})$ $-L_{i}(x)=0$.

\section{Experiment Design}

\subsection{Benchmarks}

To assess the effectiveness of SMS for the prediction of module complexity, we compare it against the perform- 
ance of two classification system benchmarks, the support vector machine and the radial basis function network.

\subsubsection{Support Vector Machine}

The support vector machine (SVM) [23,24] is an important family of supervised learning algorithms that select models that maximize the error margin of a training (design) subset. This approach has been successively used in a wide range of pattern classification problems $[25,26]$. Given a set of patterns that are assigned to one of either two classes, an SVM finds the hyperplane leaving the largest possible fraction of patterns of the same class on the same side while maximizing the distance of either class from the hyperplane. The SVM approach is usually formulated as a constrained optimization problem and solved using constrained quadratic programming.

While the original SVM approach [26] could only be used for linearly separable problems, it may be extended by employing a "kernel trick" [27] that exploits the fact that a non-linear mapping of sufficiently high dimension can project the patterns to a new parameter space in which classes can be separated by a hyperplane. In general, it cannot be determined a priori which kernel will produce good classification results for a given dataset so one must rely on heuristic (trial and error) experiments. Common kernel functions, $K(\boldsymbol{x}, \boldsymbol{y})$, for patterns, $\boldsymbol{x}, \boldsymbol{y}$, include $(\boldsymbol{x} \times \boldsymbol{y})^{\mathrm{d}},(a \boldsymbol{x} \times \boldsymbol{y}+b)^{\mathrm{d}}, \tanh (a \boldsymbol{x} \times \boldsymbol{y}+b)$, and $\exp \left(-1 / 2|\boldsymbol{x}-\boldsymbol{y}|^{2} / \sigma\right)$.

\subsubsection{Radial Basis Function Network}

A radial basis function network (RBF) [28], which may be used for pattern classification, has an internal representation of nodes that possess radial symmetry $\mathrm{f}(\boldsymbol{x})=$ $\varphi(\|\boldsymbol{x}-\boldsymbol{\mu}\|)$ where: $\boldsymbol{\mu}$ is its centre; $\|\cdot\|$ is a distance metric that determines how far an input pattern is from $\mu$; and, the transfer function, $\varphi$, must output high values when the distance from a pattern to $\boldsymbol{\mu}$ is small, and low values otherwise. While RBF train quickly [29], selecting the number of receptive fields is strictly ad hoc. If $\boldsymbol{\mu}_{i}$ is a column vector representing the centre of pattern layer node $i$ and $\sigma_{i}$ is its receptive region diameter, then $z_{i}(\boldsymbol{x})=$ $\exp \left[-\left(\boldsymbol{x}-\boldsymbol{\mu}_{i}\right)^{\mathrm{T}}\left(\boldsymbol{x}-\boldsymbol{\mu}_{i}\right) /\left(2 \sigma_{i}^{2}\right)\right.$ is the output for a given pattern, $\boldsymbol{x}$. The values $\boldsymbol{\mu}$ and $\sigma$ may analogously be viewed as the mean and standard deviation of the response curve, respectively.

The response function of an RBF node diminishes rapidly as patterns deviate from its mean. Here, the pattern layer weights are trained as well as the location and shape of the response curves. Standard k-means clustering is used to compute a $\boldsymbol{\mu}$ set. The radius of the normalized $z_{i}$ is determined by $\sigma_{i}$. If $\boldsymbol{\mu}_{i}$ is widely separated then $\sigma_{i}$ should be large to cover the gaps. If they are tightly packed then $\sigma_{i}$ should be small enough to accurately retain the distinctiveness of each receptive field. $P$-nearest neighbor is a standard heuristic algorithm used to determine $\sigma_{i}$ is. Given a receptive region's centre, $\sigma_{i}$, let $i_{1}, \cdots, i_{p}$ be the indices of the $P$ centres nearest to $\boldsymbol{\mu}_{i}$. Then $\sigma_{i}=\left(P^{-1} \Sigma_{\mathrm{p}}\left\|\boldsymbol{\mu}_{i}-\boldsymbol{\mu}_{i p}\right\|^{2}\right)^{1 / 2}$ (here, $\left.P=1\right)$.

\subsection{Measuring Performance}

Selecting a method for measuring the performance (accuracy) of a classification system is often taken for granted, which often leads to overly optimistic classification performance results. Given an $n \times n$ confusion matrix of desired versus predicted class labels, classification performance is typically measured using the observed agreement, $P_{o}=N^{-1} \Sigma_{i} n_{i i}(i=1,2, \cdots, c)$, which is the ratio of patterns that lie in regions associated with their corresponding classes to the total number of patterns regardless of where they lie $\left(n_{i i}\right.$ are the diagonal elements of the confusion matrix). While often used to measure classification performance, $P_{o}$ does not take into account the classification agreement that might be due to chance, $P_{r}=$ $N^{-2} \Sigma_{i}\left(\Sigma_{j} n_{i j} \Sigma_{j} n_{j i}\right)(i, j=1,2, \cdots, c)$ [30]. A more conservative classification performance measure is the $\kappa$ score $[31,32]$, a chance-corrected measure of agreement between the desired and predicted class assignments, $\kappa=$ $\left(P_{o}-P_{r}\right) /\left(1-P_{r}\right)$. If the classification agreement is due strictly to chance, $\kappa=0$. If the agreement is greater than chance $\kappa>0 ; \kappa=1$ indicates complete (perfect) agreement. If the agreement is less than chance then $\kappa<0$ (the minimum value depends upon the marginal distributions).

\subsection{Parameter Settings}

The following parameter values were used with the SMS algorithm: underlying classifier, LDA; frequency histogram threshold, 0.4; temperature threshold, $\mathrm{t}=0.5$; number of metric subsets, 1-11; metric subset cardinality, 1-11; validation, $b=5$; and classification performance measure, $\kappa$. For all experiments, five receptive fields were used for the RBF algorithm and the Gaussian kernel was used for SVM. Concerning SMS quadratic metric combinations, $30 \%$ were squared terms, pair-wise cross products were utilized $30 \%$ of the time, and the remaining $40 \%$ used subsets of the original metrics. For SMS, each classification experiment was allowed to run for $10^{5}$ iterations.

\section{Results and Discussion}

\subsection{Predictive Power}

Tables 1 and 2 list the breakdown of software module patterns into design $\left(\boldsymbol{X}^{D}\right)$ and validation $\left(\boldsymbol{X}^{V}\right)$ subsets of metrics for the respective datasets, DS3 and DS4, as des 
Table 1. Low/high complexity breakdown: Design and validation subsets for DS3.

\begin{tabular}{cccc}
\hline \multirow{2}{*}{$\begin{array}{c}\text { Class } \\
\text { Label }\end{array}$} & $\boldsymbol{X}^{D}$ & $\boldsymbol{X}^{V}$ & Motal \\
\cline { 2 - 4 } Low $\left(\omega_{1}\right)$ & 128 & 64 & $N_{11}=192$ \\
High $\left(\omega_{2}\right)$ & 128 & 70 & $N_{12}=198$ \\
\hline
\end{tabular}

Table 2. Low/high complexity breakdown: Design and validation subsets for DS4.

\begin{tabular}{cccc}
\hline Class & \multicolumn{3}{c}{ Metric Patterns } \\
\cline { 2 - 4 } Label & $\boldsymbol{X}^{D}$ & $\boldsymbol{X}^{V}$ & Total \\
\hline Low $\left(\omega_{1}\right)$ & 114 & 106 & $N_{21}=220$ \\
$\operatorname{High}\left(\omega_{2}\right)$ & 114 & 56 & $N_{22}=170$ \\
\hline
\end{tabular}

cribed in Section 2.3. The SMS, SVM, and RBF algorithms all use $\boldsymbol{X}^{D}$ to design their respective classification mappings and $\boldsymbol{X}^{V}$ to validate the classification performance.

Table 3 lists the mean (with standard deviations) classification performance results for DS3 with all classifiers using the validation subset of metrics, $\boldsymbol{X}^{V}$. It is clear that the SMS algorithm produced significantly better classification results than either benchmark (recall that both benchmarks used all of the original metrics). Compared against the best classifier benchmark (the SVM algorithm), SMS produced a $29 \%$ increase in the $\kappa$ score $(0.71 \pm 0.02$ versus $0.55 \pm 0.02)$ and a $10 \%$ increase in the standard $P_{o}$ agreement measure $(0.86 \pm 0.01$ versus $0.78 \pm 0.01$ ).

At this point, it is important to note the greater rate of improvement with the conservative $\kappa$ score. This demonstrates that the classification performance increase with SMS is more significant than $P_{o}$ would suggest. This is because the SMS algorithm had a concomitant improvement in both the sensitivity and specificity results. Finally, the class-wise accuracy $(0.90 \pm 0.01)$ for high $\left(\omega_{2}\right)$ complexity software modules is an excellent result as one of the motivations for this classification system is to have high predictive power for problematic software modules (that is, those considered to be of high complexity).

Table 4 lists the mean (with standard deviations) classification performance results for DS3 with all classifiers using the validation subset of metrics, $\boldsymbol{X}^{V}$. Again, SMS produced better results than the best benchmark (SVM): a $12 \%$ increase in the $\kappa$ score $(0.58 \pm 0.02$ versus $0.52 \pm$ $0.02)$ and a $4 \%$ increase for $P_{o}(0.81 \pm 0.01$ versus $0.78 \pm$ $0.01)$. It is interesting to note that the SMS $P_{o}$ values for DS3 and DS4 are similar, 0.86 and 0.81 , respectively, (only a $6 \%$ difference) but the $\kappa$ scores are sig nificantly
Table 3. DS3 Validation Subset (XV) results with $\kappa$, PO, and class-wise accuracies.

\begin{tabular}{lcccc}
\hline \multirow{2}{*}{ Classifier } & \multicolumn{4}{c}{ Accuracies } \\
\cline { 2 - 5 } & Low $\left(\omega_{1}\right)$ & High $\left(\omega_{2}\right)$ & $\kappa$ & $P_{o}$ \\
\hline SMS & $0.81 \pm 0.01$ & $0.90 \pm 0.01$ & $0.71 \pm 0.02$ & $0.86 \pm 0.01$ \\
RBF & $0.67 \pm 0.03$ & $0.81 \pm 0.01$ & $0.49 \pm 0.03$ & $0.75 \pm 0.02$ \\
SVM & $0.70 \pm 0.01$ & $0.84 \pm 0.02$ & $0.55 \pm 0.02$ & $0.78 \pm 0.01$ \\
\hline
\end{tabular}

Table 4. DS3 Validation Subset (XV) results with $\kappa$, PO, and class-wise accuracies.

\begin{tabular}{lcccc}
\hline \multirow{2}{*}{ Classifier } & \multicolumn{4}{c}{ Accuracies } \\
\cline { 2 - 5 } & Low $\left(\omega_{1}\right)$ & High $\left(\omega_{2}\right)$ & $\kappa$ & $P_{o}$ \\
\hline SMS & $0.86 \pm 0.01$ & $0.71 \pm 0.02$ & $0.58 \pm 0.02$ & $0.81 \pm 0.01$ \\
RBF & $0.80 \pm 0.01$ & $0.70 \pm 0.02$ & $0.49 \pm 0.01$ & $0.77 \pm 0.01$ \\
SVM & $0.81 \pm 0.01$ & $0.71 \pm 0.02$ & $0.52 \pm 0.02$ & $0.78 \pm 0.01$ \\
\hline
\end{tabular}

different, 0.71 and 0.58 , respectively (a $22 \%$ difference). This is evidenced by the poorer balance between the sensitivity and specificity in the case of DS4. Again, while $P_{o}$ suggests little difference in the classification performance results using the different datasets, $\kappa$ strongly suggests that DS3 is a much better delineated dataset.

\subsection{Predictive Software Metrics}

In the case of DS3, two software metric subsets were selected as being highly discriminatory. One subset used eight of the original software metrics: $\mathrm{C} 1, \mathrm{C} 2, \mathrm{C} 3, \mathrm{C} 4, \mathrm{P} 1$, $\mathrm{P} 2, \mathrm{P} 3$, and $\mathrm{F} 1$. The other subset was a pair-wise cross product of the metrics $\mathrm{C} 1, \mathrm{C} 2, \mathrm{C} 3$, and $\mathrm{C} 4$ with $\mathrm{C} 1, \mathrm{C} 2$, and C3. In the case of DS4, two metric subsets were also selected. The first used ten of the original software metrics excluding only F2. The other metric subset was the squared values of all software metrics except F2 and F1. Both experiments demonstrate that quadratic combinations of these software metrics effect greater predictive power than any combination of the original software metrics.

\section{Conclusions}

The stochastic metric selection algorithm has been shown to be an effective strategy for the prediction of software module complexity, as expressed by module change count, given a set of software metrics. Compared to the conventional classification system benchmarks, this strategy produced significantly better overall classification results, as well as improved sensitivity and specificity, using only a subset of the original metrics. Finally, it was particularly effective in predicting the occurrence of high 
complexity modules, which is an important characteristic for software project managers and program developers when maintaining and updating software modules.

\section{Acknowledgements}

The author would like to thank A. Demko and C. Wiebe for their valuable contributions in implementing the stochastic metric selection algorithm in Scopira. The author would also like to thank M. R. Lyu for making the MIS dataset available for this pattern classification investigation.

This work was supported in part by the Natural Sciences and Engineering Research Council of Canada (NSERC).

\section{REFERENCES}

[1] S. R. Chidamber and C. F. Kemerer, "A Metrics Suite for Object-Oriented Design," IEEE Transactions on Software Engineering, Vol. 20, No. 6, 1994, pp. 476-493. doi: $10.1109 / 32.295895$

[2] B. A. Kitchenham, R. T. Hughes and S. G. Kinkman, "Modeling Software Measurement Data," IEEE Transactions on Software Engineering, Vol. 27, No. 9, 2001, pp. 788-804. doi:10.1109/32.950316

[3] N. E. Fenton and A. A. Kaposi, "Metrics and Software Structure," Information and Software Technology, Vol. 29, No. 6, 1987, pp. 301-320. doi:10.1016/0950-5849(87)90029-2

[4] N. E. Fenton and M. Neil, "A Critique of Software Defect Prediction Models," IEEE Transactions on Software Engineering, Vol. 25, No. 5, 1999, pp. 675-689. doi: $10.1109 / 32.815326$

[5] G. Poels and G. Dedene, "Distance-Based Software Measurement: Necessary and Sufficient Properties for Software Measures," Information and Software Technology, Vol. 42, No. 1, 2000, pp. 35-46.

[6] E. J. Weyuker, "Evaluating Software Complexity Measures," IEEE Transactions on Software Engineering, Vol. 14, No. 9, 1988, pp. 1357-1365. doi:10.1109/32.6178

[7] M. Reformat, W. Pedrycz and N. J. Pizzi, "Software Quality Analysis with the Use of Computational Intelligence," Information and Software Technology, Vol. 45, No. 7, 2003, pp. 405-417. doi:10.1016/S0950-5849(03)00012-0

[8] G. A. F. Seber, "Multivariate Observations," John Wiley \& Sons Ltd., Hoboken, 1984. doi: $10.1002 / 9780470316641$

[9] R. O. Duda, P. E. Hart and D. G. Stork, "Pattern Classification," 2nd Edition, Wiley-Interscience, New York, 2000.

[10] R. Gnanadesikan, "Methods for Statistical Data Analysis of Multivariate Observations," 2nd Edition, John Wiley \& Sons Ltd., New York, 1997. doi:10.1002/9781118032671

[11] N. J. Pizzi and W. Pedrycz, "Effective Classification Using Feature Selection and Fuzzy Integration," Fuzzy Sets and Systems, Vol. 159, No. 21, 2008, pp. 2859-2872. doi:10.1016/j.fss.2008.03.015

[12] M. R. Lyu, "Data Directory in the CD-ROM," http://www.cse.cuhk.edu.hk/ lyu/book/reliability/data.ht $\mathrm{ml}$

[13] J. C. Munson and T. M. Khoshgofthaar, "Software Metrics for Software Reliability Assessment," In: M. R. Lyu, Ed., Handbook of Software Reliability Engineering, McGraw-Hill, New York, 1996, pp. 493-529.

[14] R. Lind and K. Vairavan, “An Experimental Investigation of Software Metrics and Their Relationship to Software Development Effort," IEEE Transactions on Software Engineering, Vol. 15, No. 5, 1989, pp. 649-653. doi: $10.1109 / 32.24715$

[15] R. S. Pressman and R. Pressman, "Software Engineering: A Practitioner's Approach," McGraw-Hill, New York, 2000.

[16] N. E. Fenton and S. L. Pfleeger, "Software Metrics: A Rigorous and Practical Approach," PWS Publishing, Boston, 1997.

[17] J. F. Peters and W. Pedrycz, "Software Engineering: An Engineering Approach," John Wiley \& Sons Ltd., Hoboken, 1999.

[18] M. H. Halstead, "Elements of Software Science," Elsevier, New York, 1977.

[19] T. J. McCabe, “A Complexity Metric," IEEE Transactions on Software Engineering, Vol. 2, No. 4, 1976, pp. 308-320. doi:10.1109/TSE.1976.233837

[20] E. K. Tang, P. N. Suganthan and X. Yao, "Gene Selection Algorithms for Microarray Data Based on Least Square Support Vector Machine," BMC Bioinformatics, Vol. 7, No. 95, 2006. doi:10.1186/1471-2105-7-95

[21] Q. Liu, A. Sung, Z. Chen and J. Xu, "Feature Mining and Pattern Classification for LSB Matching Steganography in Grayscale Images," Pattern Recognition, Vol. 41, No. 1, 2008, pp. 56-66.

[22] N. Kasabov and Q. Song, "DENFIS: Dynamic Evolving Neural-Fuzzy Inference System and Its Application for Time-Series Prediction," IEEE Transactions on Fuzzy Systems, Vol. 10, No. 2, 2002, pp. 144-154. doi:10.1109/91.995117

[23] B. Schölkopf and A. J. Smola, "Learning with Kernels: Support Vector Machines, Regularization, Optimization, and Beyond," MIT Press, Cambridge, 2002.

[24] V. Vapnik, "Statistical Learning Theory," John Wiley \& Sons Ltd., New York, 1998.

[25] L. Wang, "Support Vector Machines: Theory and Applications," Springer-Verlag, Berlin, 2005.

[26] V. Vapnik and A. Lerner, "Pattern Recognition Using Generalized Portrait Method," Automation and Remote Control, Vol. 24, 1963, pp. 774-780.

[27] W. H. Press, S. A. Teukolsky, W. T. Vetterling and B. P. Flannery, "Numerical Recipes: The Art of Scientific Computing," 3rd Edition, Cambridge University Press, Cambridge, 2007.

[28] J. Moody and C. J. Darken, "Fast Learning Networks of 
Locally-Tuned Processing Units," Neural Computation, Vol. 1, No. 2, 1989, pp. 281-294.

[29] S. M. Weiss and C. A. Kulikowski, "Computer Systems that Learn: Classification and Prediction Methods from Statistics, Neural Nets, Machine Learning and Expert Systems," Morgan Kaufmann Publishing, San Mateo, 1991.

[30] B. S. Everitt, "Moments of the Statistics Kappa and Weighted Kappa," British Journal of Mathematical and Statistical Psychology, Vol. 21, 1968, pp. 97-103.
[31] J. L. Fleiss, "Measuring Agreement between Two Judges on the Presence or Absence of a Trait," Biometrics, Vol. 31, No. 3, 1975, pp. 651-659. doi:10.2307/2529549

[32] T. McGinn, P. C. Wyer, T. B. Newman, S. Keitz, R. Leipzig and G. Guyatt, "Tips for Learners of Evidence-Based Medicine: 3. Measures of Observer Variability (Kappa Statistic)," Canadian Medical Association Journal, Vol. 171, No. 11, 2004, pp. 1369-1373. doi:10.1503/cmaj.1031981 